\title{
Mapeamento da produção científica sobre memória organizacional e ontologias
}

\section{Airton Zancanaro}

Doutorando do Programa de Pós-Graduação em Engenharia e Gestão do Conhecimento (EGC) na Universidade Federal de Santa Catarina (UFSC). Bolsista CAPES

Julio Graeff Erpen

Doutorando do Programa de Pós-Graduação em Engenharia e Gestão do Conhecimento (EGC) na Universidade Federal de Santa Catarina (UFSC)

Jane Lucia S. Santos

Doutoranda do Programa de Pós-Graduação em Engenharia e Gestão do Conhecimento (EGC) na Universidade Federal de Santa Catarina (UFSC) Bolsista CAPES

Andrea Valeria Steil

Professora do Programa de Pós-Graduação em Engenharia e Gestão doConhecimento na Universidade Federal de Santa Catarina - EGC / UFSC e do Departamento de Psicologia / UFSC

José Leomar Todesco

Professor do Programa de Pós-Graduação em Engenharia e Gestão do Conhecimento na Universidade Federal de Santa Catarina - EGC / UFSC

Memória organizacional e gestão do conhecimento são temas interligados que têm crescido em importância para pesquisadores e organizações, ao longo dos anos 2000. A literatura aponta as ontologias como importantes métodos que podem ser aplicados para formalizar e representar a memória organizacional. Entretanto, existe um hiato quanto à investigação das pesquisas desenvolvidas na intersecção dessas duas áreas. Este artigo objetiva mapear o perfil e as características das publicações relacionadas à memória organizacional e às ontologias, por meio de técnicas bibliométricas. Foram localizados 39 trabalhos, os quais foram escritos por 85 autores, 
vinculados a 40 instituições de 18 países diferentes. Para a elaboração desses trabalhos, os autores utilizaram 845 referências e 117 palavras-chave, dentre as quais se destacam Knowledge management, e-Learning e Organizationallearning. Em suma, este estudo possibilita a visualização de padrões e tendências da literatura científica, mostrando quais pesquisas podem ser promissoras no campo de memória organizacional e ontologias.

Palavras-chave: Memória organizacional; Ontologias; Bibliometria; Produção científica.

\section{Mapping of the scientific production on organizational memory and ontologies}

Organizational Memory and Knowledge management are interconnected themes that have grown up in importance to researchers and organizations over the years 2000. The literature has indicated Ontologies as important methods that can be applied to formalize and represent organizational memory. However, there is a knowledge gap at the intersection of these two areas. This article aimed to map the publications related to organizational memory and ontologies through bibliometric techniques. 39 papers were identified, which were written by 85 authors, linked to 40 institutions from 18 countries. For the elaboration of their work, the authors used 845 references and 117 keywords, among which are highlighted Knowledge Management, e-Learning and Organizational Learning. In short, this work enabled the visualization of patterns and trends of the scientific literature and shows that research may be promising in the field of organizational memory and ontologies.

Keywords: Organizational memory; Ontologies; Bibliometrics; Scientific production.

Recebido em 04.09.2012 Aceito em 08.02.2013

\section{Introdução}

Memória organizacional e gestão do conhecimento são temas interligados que têm crescido em importância para pesquisadores e 
organizações, ao longo dos anos 2000 (NEVO; WAND, 2005; SANTOS; URIONA-MALDONADO; MACEDO DOS SANTOS, 2011). Diversos estudiosos ressaltam o aumento das atenções voltadas à memória organizacional no âmbito acadêmico (tais como STEIN, 1995; LEHNER; MAIER, 2000; ANDERSON; SUN, 2010), mas poucos procuram retratar amplamente as características da produção científica nesse campo e suas relações com temáticas associadas.

Por meio de um estudo bibliométrico, Santos, Uriona-Maldonado e Macedo dos Santos (2011) mapearam o perfil e as características do campo das pesquisas sobre memória organizacional. Os referidos autores identificaram três grandes grupos de artigos. O primeiro grupo adota uma abordagem tecnológica para estudar memória organizacional. Parte dos artigos desse grupo investiga sistemas de informação baseados em computador (computer-basedinformation systems), tratando-os como repositórios de conhecimentos (por exemplo, o artigo de KANKANHALLI; TAN; WEI, 2005). O segundo grupo de trabalhos, como o de Abecker e outros (1998), estuda as tecnologias colaborativas (collaborativetechnologies) e os sistemas de apoio à decisão (decisionsupport systems), tratando-os como sistemas que se baseiam em conhecimento. Por fim, o terceiro grupo de estudos enfoca o desenvolvimento e a aplicação de ontologias (ontologies), principalmente para representar e organizar o conhecimento organizacional, por exemplo, os trabalhos de Ju (2006) e Weinberger, Te'Eni e Frank (2008).

Entretanto, ao traçar um quadro geral das pesquisas sobre memória organizacional, Santos, Uriona-Maldonado e Macedo dos Santos (2011) não retratam, de modo específico, os perfis dos estudos que investigam os artefatos tecnológicos nesse campo de pesquisa.

O campo de pesquisa em memória organizacional tem sido bastante influenciado pelo trabalho seminal de Walsh e Ungson (1991), no qual os autores desenvolvem seu argumento a partir da crença de que a estrutura da memória organizacional é composta por um conjunto de repositórios de armazenamento (storagebins). Essa abordagem se fortaleceu e a compreensão de que o conhecimento organizacional é armazenado e preservado em vários repositórios tornou-se fundamental para os estudos da memória organizacional (STEIL; SANTOS, 2012). Talvez, por isso, uma parte significativa dos trabalhos sobre o tema, tais como os artigos de Olivera (2000) e Cardenas e Spinola (2010), tenha buscado identificar esses diferentes repositórios e os meios que podem apoiar os processos de aquisição, armazenamento, acesso e uso de conhecimentos nas organizações.

Nesse contexto, as ontologias são citadas por alguns pesquisadores como sendo relevantes métodos que podem ser empregados para formalizar e representar o conhecimento organizacional, com a finalidade de recuperá-lo e aplicá-lo posteriormente (BORST, 1997; RAUTENBERG; TODESCO; GAUTHIER, 2009) e que o desenvolvimento e a aplicação de ontologias são notavelmente relevantes para o campo da aprendizagem 
organizacional (VALASKI; MALUCELLI; REINEHR, 2012) e da memória organizacional (MELGAR SASIETA; BEPPLER; PACHECO, 2011).

O conceito do termo "ontologias" foi atribuído inicialmente a Gruber (1993a, p.1), que o define como "uma especificação explícita de uma conceitualização". Borst (1997, p.12), por sua vez, complementou essa definição dizendo que ontologia é a "especificação formal e explícita de uma conceitualização compartilhada", ou seja, uma ontologia modela parte do mundo e pode ser descrita conforme taxonomias e axiomas (RAUTENBERG; TODESCO; GAUTHIER, 2009) e, ainda, apoia as atividades de compartilhar conhecimentos (GUARINO, 1995). Pesquisas, como a de Dias e Costa (2011), mapeiam, dentre outros assuntos, os diversos autores que conceituam ontologias e trazem, por exemplo, os trabalhos de Gruber (1995) e Uschold e Gruninger (1996), como os de referências na área.

A utilização de ontologias tem sido apontada como uma tendência em diversas áreas e contextos (MUSEN, 2002) e a motivação para o seu desenvolvimento é associada aos vários benefícios que podem ser obtidos com o seu uso (VALASKI; MALUCELLI; REINEHR, 2012). Como destacam Valaski, Malucelli e Reinehr (2012), existe um grande interesse relacionado à construção de ontologias, no entanto, parece haver escassez de pesquisas realizadas nessa área. Acredita-se que um levantamento da literatura sobre memória organizacional e ontologias pode contribuir para identificar as intersecções existentes entre essas duas áreas de pesquisa, de modo a: (i) relacionar os basilares teóricos nos quais se fundamentam os estudos que buscam estabelecer algum tipo de interface entre os dois temas; e (ii) localizar os principais autores e grupos de pesquisas mais representativos que têm desenvolvido estudos nessa direção e as temáticas associadas à memória organizacional e ontologias.

Por meio da aplicação de técnicas bibliométricas, este artigo tem por objetivo mapear o perfil e as características das publicações que têm estudado, conjuntamente, memória organizacional e ontologias. Apresenta-se, na seção 2, o detalhamento dos procedimentos metodológicos utilizados para o desenvolvimento da pesquisa bibliométrica. Na seção 3, são descritos e discutidos os principais resultados. E, na seção 4, são descritas as considerações finais do artigo, seguidas pela lista de referências.

\section{Procedimentos metodológicos para realização da pesquisa bibliométrica}

Ao considerar que a análise da produção científica sobre determinado tema é sensível às particularidades e características da área (HERCULANO; NOBERTO, 2011), este trabalho adotou uma combinação de diferentes técnicas bibliométricas e seguiu algumas recomendações e descrições sugeridas por Marcias-Chapula(1998), Guedes e Borschiver (2005) e Kobashi e Santos (2006). Para realizar este estudo bibliométrico, foram desenvolvidas duas fases de pesquisa: 1) coleta, filtragem e 
padronização dos dados; e 2) análise e síntese dos dados coletados e elaboração do documento final. Essas fases, juntamente com as etapas correspondentes, estão representadas na Figura 1.

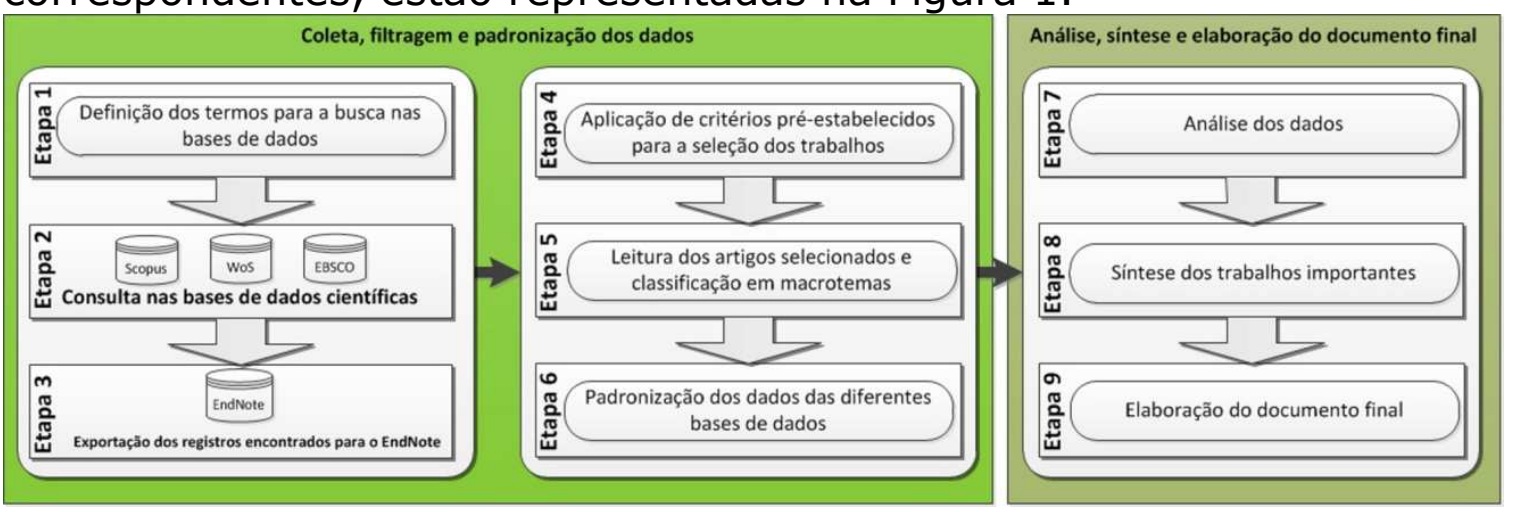

Figura 1 - Fases e etapas da pesquisa bibliográfica realizada nas bases de dados Web of Science, Scopus e EBSCO para os termos "memória organizacional" e "ontologias".

Fonte: Dados da pesquisa.

Nos próximos subitens, está descrito como essas etapas foram desenvolvidas.

\subsection{Etapa 1: definição dos termos para as buscas nas bases de dados}

A associação dos termos "memória organizacional" e "ontologias" tem por objetivo identificar quais as áreas de estudo e as linhas de pesquisa dos trabalhos indexados nas bases de dados científicas internacionais. Para a realização das buscas, foram utilizados os termos em inglês: Organi?ationalMemor* andOntolog*. O símbolo "?" representa a busca por qualquer caractere nessa posição, permite incluir termos do inglês britânico (organisational) e norte-americano (organizational). Já o símbolo "*", permite localizar variações das palavras pesquisadas, como, por exemplo, o seu plural - com isto foi possível incluir na consulta termos como memory, memories, ontology e ontologies.

\subsection{Etapa 2: consulta nas bases de dados científicas}

As buscas foram realizadas em três bases de dados internacionais: a Web of Science (WoS), a Scopus e a EBSCO. A primeira indexa mais de 12.700 periódicos nas áreas de ciências, ciências sociais, artes e humanidades (LOPES et al., 2012) e é considerada uma das bases mais relevantes e mais usadas em estudos bibliométricos (CROSSAN; APAYDIN, 2010; BRAMBILLA; STUMPUF, 2012). A segunda é uma base científica mundial que indexa artigos desde 1960, com mais de 18.500 periódicos (OLIVEIRA; GRACIO, 2011) e que cobre áreas do conhecimento, como a engenharia, as ciências sociais, a economia e as ciências gerais 
(MESQUITA et al., 2006). A última base indexa mais de 7.900 periódicos de texto completo e inclui mais de 6.800 periódicos revisados por pares.

A seleção dessas bases de dados deu-se por serem bases multidisciplinares reconhecidas pela comunidade acadêmica internacional (ALMEIDA, 2006), possuírem registros sobre o tema pesquisado e permitirem exportar os dados para o gerenciador de referências bibliográficas EndNote ${ }^{1}$.

Devido às características próprias de cada base de dados principalmente quanto à sua estrutura e à forma de indexar as publicações - foram utilizadas diferentes estratégias de busca para cada uma delas. Na WoS, os termos definidos na etapa 1 foram pesquisados nos campos que compõem o título, as palavras-chave e o resumo das publicações, denominado de Topic. Na Scopus, esse campo é denominado de article, title, abstract, keywords. $\mathrm{E}$, na EBSCO, por não permitir a busca em alguns campos mais importantes, optou-se por deixar a opção "selecionar um campo (opcional)". Nesse caso, os termos de busca foram localizados em todos os campos disponíveis na base. Não houve restrição temporal inicial ou de idioma na consulta às bases de dados. Foram considerados todos os registros existentes até dezembro de 2011.

\subsection{Etapa 3: exportação dos registros encontrados para o EndNote}

Com os resultados iniciais das consultas, foi possível gerar arquivos com os principais dados bibliométricos dos trabalhos, como título, autores, ano e local de publicação (entre outras). Esses dados foram importados para o EndNote, que gerou um único conjunto de artigos.

\subsection{Etapa 4: aplicação de critérios preestabelecidos para a seleção dos trabalhos}

No EndNote, foram aplicados os seguinte critérios para a seleção dos trabalhos: retirada de artigos sem autoria; remoção de artigos duplicados (artigos indexados em mais de uma base de dados); e exclusão de artigos sem textos completos disponíveis para download gratuitamente.

\subsection{Etapa 5: classificação dos artigos em macrotemas (temáticas de pesquisa)}

Nesta etapa foi lido e analisado o texto completo dos artigos selecionados. Para realizar essa análise, foram adotados os seguintes procedimentos: a) os pesquisadores adotaram o softwareCiteSpace ${ }^{2}$ para

\footnotetext{
EndNote é um software criado pela Thompson Reuters que permite pesquisar em bases de dados bibliográficas online, organizar as referências, imagens, arquivos .pdf e gerenciar a bibliografia no editor de texto (Disponível em: <http://www.endnote.com>. Acesso em: 23 jan. 2013)

${ }^{2}$ CiteSpace foi desenvolvido por Chaomei Chen e encontra-se disponível gratuitamente para ser acessado. 0 objetivo do software é a visualização progressiva do conhecimento referente a um determinado domínio, através de ferramentas que facilitam a compreensão e a interpretação dos padrões em redes em clusters (Disponível em: <http://cluster.cis.drexel.edu/ cchen/citespace>. Acesso em: 23 jan. 2013).
} 
gerar uma lista inicial de temas identificados a partir da similaridade dos títulos, palavras-chave e resumos; b) essa lista foi utilizada como referência, para classificar os artigos de acordo com a sua temática principal; c) três dos pesquisadores leram os 39 artigos, associaram-nos e classificaram-nos em macrotemas e com uma (ou mais) temática que estava na lista e, quando não era possível classificar o trabalho em uma dessas temáticas, o pesquisador poderia apontar outra na qual o artigo se enquadrasse; e d) esses pesquisadores realizaram duas reuniões, entre si, para discutir a classificação dos artigos por macrotemas e temática.

\subsection{Etapa 6: padronização dos dados das diferentes bases}

Pelo fato de a consulta ter sido realizada em três bases de dados distintas, com diferentes critérios de apresentação dos campos, como a exibição do nome dos autores, gerou a necessidade de padronizar os dados. Na Base Scopus, por exemplo, a autoria é identificada pelo sobrenome e pela letra inicial do nome. Já na WoS, o nome é escrito por extenso. Além disso, informações referentes ao vínculo institucional dos autores, às referências e às palavras-chave, muitas vezes, não estavam disponíveis nessas bases, por isso foi necessário coletar diretamente no documento. Assim, uma nova base foi criada com auxilio do Microsoft Access, e o preenchimento das informações de cada artigo foi efetuado manualmente: as informações dos autores e coautores (sobrenome, nome, vínculo institucional e a local da instituição), dos artigos (ano de publicação, idioma, tipo de documento, periódico, resumo e principais temáticas), das referências citadas (autor, ano, título do trabalho) e das palavras-chave.

\subsection{Etapa 7: análise dos dados}

Com os dados padronizados, foram realizadas as consultas e a contagem de frequência. Esse registro possibilitou gerar análises bibliométricas, como quantidade de publicações por ano, tipos e fontes de publicação, autores e instituições de vínculo e seus correspondentes países (com o objetivo de identificar os principais autores e grupos de pesquisas mais representativos na área deste trabalho), palavras-chave, temáticas abordadas, análise das referências mais citadas (para identificar os basilares teóricos nos quais se fundamentam os trabalhos que buscam estabelecer algum tipo de interface entre os dois temas).

\subsection{Etapa 8: síntese dos trabalhos importantes}

Pelo referencial bibliográfico dos artigos localizados nas bases de dados, identificaram-se as referências mais citadas pelos trabalhos sobre memória organizacional e ontologias. Foram identificadas, analisadas e sintetizadas as seis obras mais citadas. 


\subsection{Etapa 9: elaboração do documento final}

De posse das informações padronizadas referentes aos trabalhos selecionados neste estudo, foi possível elaborar o documento com as contextualizações, descrições e análises das pesquisas, e, posteriormente, essas informações foram organizadas no formato de artigo científico.

Nos próximos itens, são descritas as análises e as discussões dos resultados obtidos por meio do desenvolvimento de cada uma das etapas descritas anteriormente.

\section{Resultados}

Nesta seção, apresentam-se os resultados das análises e sínteses das informações selecionados a partir das buscas realizadas nas bases de dados EBSCO, Scopus e WoS.

\subsection{Dados bibliométricos gerais sobre as pesquisas de memória organizacional e ontologias}

A pesquisa bibliométrica, realizada nas bases de dados, permitiu localizar 18 registros na EBSCO, 45 na Scopus e 17 na WoS, e resultou em um total de 80 trabalhos. A Tabela 1 ilustra o processo para a seleção dos artigos finais para a análise.

Tabela 1 - Número de publicações selecionadas nas bases de dados WoS, Scopus e EBSCO para os termos "memória organizacional" e "ontologias"

\begin{tabular}{llllll}
\hline $\begin{array}{l}\text { Base de } \\
\text { dados }\end{array}$ & $\begin{array}{l}\text { Publicaçõ } \\
\text { es } \\
\text { localizada } \\
\text { s }\end{array}$ & $\begin{array}{l}\text { Exclusão das } \\
\text { publicações sem } \\
\text { autoria disponível }\end{array}$ & $\begin{array}{l}\text { Exclusão } \\
\text { das } \\
\text { publicações } \\
\text { repetidas }\end{array}$ & $\begin{array}{l}\text { Exclusão das } \\
\text { publicações sem } \\
\text { texto completo } \\
\text { disponível }\end{array}$ & $\begin{array}{l}\text { Total de } \\
\text { publicaçõ } \\
\text { es } \\
\text { seleciona } \\
\text { das }\end{array}$ \\
\hline EBSCO & 18 & 0 & 8 & 3 & 7 \\
SCOPU & 45 & 4 & 9 & 6 & 26 \\
S & & 0 & 11 & 0 & 6 \\
WoS & 17 & $\mathbf{4}$ & $\mathbf{2 8}$ & $\mathbf{9}$ & $\mathbf{3 9}$ \\
Total & $\mathbf{8 0}$ & $\mathbf{4}$ & & & \\
\hline
\end{tabular}

Fonte: Dados da pesquisa.

Entre os 39 trabalhos selecionados: 18 foram publicados em anais de eventos e 21, artigos indexados em periódicos científicos. Esses trabalhos foram escritos por 85 autores, vinculados a 40 instituições de 18 países diferentes. Os autores utilizaram 117 palavras-chave e 845 referências (média de 19 referências citadas por publicação). A Tabela 2 sintetiza os dados bibliográficos gerais da pesquisa. 
Tabela 2 - Dados bibliográficos gerais das publicações selecionadas nas bases de dados WoS, Scopus e EBSCO para os termos "memória organizacional" e "ontologias"

\begin{tabular}{ll}
\hline Dados bibliográficos & Frequência \\
\hline Publicações & 39 \\
Fontes de publicação & 35 \\
Autores & 85 \\
Instituições & 40 \\
Países & 18 \\
Palavras-chave & 117 \\
Referências citadas & 845 \\
\hline
\end{tabular}

Fonte: Dados da pesquisa.

Nos próximos itens, estão descritas as análises enfocadas em cada um dos dados bibliométricos apresentados na Tabela 2.

\subsection{Publicações por ano}

A série histórica apontou que grande parte dos artigos sobre a temática enfocada por este trabalho foi publicada em 2004 e 2007, ambos com sete publicações cada. Identificou-se que, em 2004, os trabalhos foram escritos por nove autores franceses, quatro brasileiros, três mexicanos, um alemão, um estadunidense e um britânico. Em 2007, autores de outras nações surgiram na lista: cinco da Polônia; três do Canadá; dois da Argélia, dos Estados Unidos e de Taiwan; um da China e da Inglaterra.

Um dos primeiros artigos identificados nesta pesquisa, que associou os termos "Memória Organizacional" e "ontologias", foi publicado em 1999, com o título Knowledgeas a social medium, escrito por Keiichi Nakata. Esse autor estava vinculado ao GermanNationalResearch Center for Information Technology, localizado na Alemanha e o seu artigo traz discussões sobre o conhecimento social nos contextos de grupos e comunidades; aborda, também, as ontologias como forma de capturar os interesses pessoais em campos como o da memória organizacional.

Inversamente, os três trabalhos mais recentes, publicados em 2011, são: a) Anontologybasedarchitecturetosupporttheknowledge management in highereducation, de Laoufi e colaboradores (2011), aborda uma proposta baseada no e-Learning, para estabelecer a memória organizacional do capital científico, técnico e administrativo da Universidade de IBN ZOHR, em Agadir, Marrocos. O repositório de conhecimento de recursos criado pelos autores inclui documentos administrativos, cursos de formação, livros eletrônicos, entre outros, que são indexados e validados por meio de uma ontologia; b) Anontologydrivendocumentretrievalstrategy for organizationalknowledge management systems, Toledo e outros (2011) propõem uma arquitetura de memória organizacional, utilizando ontologias de domínio como forma de recuperação de informação; e c) OMCCAAF conceptualizingaftaxonomyand thesaurus construction, de Anica-Popa (2011), aborda um estudo sobre as taxonomias em contabilidade e 
finanças e as etapas para a construção de tesauros, trazendo a sua importância para a modelagem da memória organizacional.

\subsection{Principais fontes de publicações}

Os artigos foram originários de 35 fontes diferentes. Destas, apenas quatro se repetiram. Os resultados são apresentados na Tabela 3.

Tabela 3 - Principais fontes de publicações nas bases de dados WoS, Scopus e EBSCO para os termos "memória organizacional" e "ontologias"

\begin{tabular}{|c|c|c|c|c|c|c|}
\hline Fontes de publicação & Qtd & ISSN & Tipo & $\begin{array}{l}\text { Fator } \\
\text { de } \\
\text { impacto }\end{array}$ & $\begin{array}{l}\text { Ano da } \\
\text { publicaç } \\
\text { ão }\end{array}$ & Editora \\
\hline $\begin{array}{l}\text { Lecture Notes in Computer } \\
\text { Science }\end{array}$ & 2 & $\begin{array}{l}0302- \\
9743\end{array}$ & $\begin{array}{l}\text { ConferenceP } \\
\text { aper }\end{array}$ & - & $\begin{array}{l}2006 \text { e } \\
2008\end{array}$ & $\begin{array}{l}\text { Springe } \\
r\end{array}$ \\
\hline $\begin{array}{l}\text { Expert Systems } \\
\text { withApplications }\end{array}$ & 2 & $\begin{array}{l}0957- \\
4174\end{array}$ & $\begin{array}{l}\text { Journal } \\
\text { Article }\end{array}$ & 1.924 & $\begin{array}{l}2002 \text { e } \\
2004\end{array}$ & Elsevier \\
\hline $\begin{array}{l}\text { Lecture Notes in Artificial } \\
\text { Intelligence }\end{array}$ & 2 & $\begin{array}{l}0302- \\
9743\end{array}$ & $\begin{array}{l}\text { ConferenceP } \\
\text { aper }\end{array}$ & - & 2004 & $\begin{array}{l}\text { Springe } \\
r\end{array}$ \\
\hline $\begin{array}{l}\text { Journal of the American } \\
\text { Society for Information } \\
\text { Science and Technology }\end{array}$ & 2 & $\begin{array}{l}1532- \\
2890\end{array}$ & $\begin{array}{l}\text { Journal } \\
\text { Article }\end{array}$ & 2.137 & $\begin{array}{l}2008 \text { e } \\
2009\end{array}$ & Wiley \\
\hline
\end{tabular}

Fonte: Dados da pesquisa.

Cada uma dessas fontes de publicações tem dois trabalhos publicados sobre memória organizacional e ontologias. Vale mencionar que as fontes de publicações Lecture Notes in Computer Science e Lecture Notes in Artificial Intelligence possuem o mesmo ISSN. Isso ocorreu porque a segunda fonte foi uma subsérie da primeira, estabelecida nos anos 1980, com foco em inteligência artificial.

\subsection{Principais autores, instituições e países}

Das publicações analisadas, 27 foram escritas por sete autores, as quais são relacionadas na Tabela 4, juntamente com o respectivo número de publicações, o vínculo institucional, a cidade e o país.

Por meio da análise das autorias dos 39 trabalhos selecionados, foi possível identificar a existência de 27 clusters de grupos de pesquisas nos temas memória organizacional e ontologias. O grupo (rede de pesquisa) mais expressivo em quantidade de publicações é o formado pela autora Marie-Hélène Abel, do Laboratório Heudiasyc (Métodos Heurísticos e Diagnósticos de Sistemas Complexos) da Universidade de Tecnologia de Compiègne, na França. Essa rede de pesquisa era composta por nove autores, entre os quais Marie-Hélène Abel era o principal hub (vértice central), ou seja, a pesquisadora, a partir da qual é possível chegar a todos os demais integrantes da rede analisada (BALANCIERI, 2004). 
Tabela 4 -Autores com maior número de publicações, vínculo institucional, cidade e país para artigos selecionados nas bases de dados WoS, Scopus e EBSCO para os termos "memória organizacional" e "ontologias"

\begin{tabular}{|c|c|c|c|c|}
\hline Autor & $\begin{array}{l}\text { Número de } \\
\text { publicações }\end{array}$ & Vínculo institucional & Cidade & País \\
\hline Abel, M. H. & 6 & University of Technology of Compiègne & Compiègne & França \\
\hline Moulin, C. & 4 & University of Technology of Compiègne & Compiègne & França \\
\hline Nkambou, R. & 4 & Universityof Quebec & Montreal & Canadá \\
\hline Zouaq, A. & 4 & Universityof Montreal & Montreal & Canadá \\
\hline Lenne, D. & 3 & University of Technology of Compiègne & Compiègne & França \\
\hline Kitowski, J. & 3 & $\begin{array}{l}\text { AGH University of Science and } \\
\text { Technology }\end{array}$ & Cracow & Polônia \\
\hline Frasson, C. & 3 & Universityof Montreal & Montreal & Canadá \\
\hline
\end{tabular}

Fonte: Dados da pesquisa.

Percebe-se que Marie-Hélène trabalhou ativamente como autora sobre tema memória organizacional para e-Learning, com aplicação de ontologias, entre 2003 e 2009. Em 2011, suas publicações foram direcionadas a temas como gestão do conhecimento em sistemas de transporte ferroviário (como pode ser observado em PENCIUC; ABEL; ABBEELE, 2011).

Também, foram identificadas as principais instituições de pesquisa com publicações a respeito do tema analisado (40 instituições). Dentre elas, as mais produtivas quanto ao número de publicações foram sete: duas no Canadá e uma na França, Alemanha, Polônia, Argélia e uma na Argentina (Tabela 5).

Tabela 5 - Quantidade de publicação e percetual por Instituições para artigos selecionados nas bases de dados WoS, Scopus e EBSCO para os termos "memória organizacional" e "ontologias"

\begin{tabular}{lll}
\hline Instituições & Qtde. & $\%$ \\
\hline 1. University of Technology of Compiègne (França) & 6 & 10,71 \\
2. University of Quebec (Canadá) & 4 & 7,14 \\
3. University of Montreal (Canadá) & 4 & 7,14 \\
4. AGH University of Science and Technology (Polonia) & 4 & 5,36 \\
5. German Research Center for Artificial Intelligence & 2 & 3,57 \\
(Alemanha) & & \\
6. National Institute of Computer Sciences (Argélia) & 2 & 3,57 \\
7. INGAR - CONICET (Argentina) & 2 & 3,57 \\
Todas as outras & 33 & 58,93 \\
\hline
\end{tabular}

Fonte: Dados da pesquisa.

Abel, juntamente com seu grupo de pesquisa, auxiliou a Universidade Tecnológica de Compiègne a ficar no topo da lista das universidades mais produtivas em relação a trabalhos sobre memória organizacional e ontologias. Essa universidade representa mais de $10 \%$ das publicações localizadas sobre o tema. 


\subsection{Principais palavras-chave e temáticas abordadas pelos trabalhos}

Os trabalhos analisados utilizaram 177 palavras-chave. As principais foram: Ontology (ontologia), utilizada em 19 trabalhos;

Organizationalmemory (memória organizacional), com 15 ocorrências; Knowledge management (gestão do conhecimento), com 14 ocorrências; ee-Learning e Organizationallearning (aprendizagem organizacional), com cinco ocorrências cada.

Conforme ilustra a Figura 2 (nuvem de termos), o termo Knowledge (conhecimento) aparece em destaque e os autores o utilizam junto com outras palavras, principalmente com: management (gestão), engineering (engenharia), acquisition (aquisição), creation (criação), representation (representação), retrieval (recuperação), sharing (compartilhamento) e discovery (descoberta). Já o termo ontology (ontologia) é geralmente associado aos termos learning (aprendizagem), matching (combinação), similarity (similaridade) e store (armazenar). Com isso, observou-se que os trabalhos que exploraram o tema memória organizacional e ontologias foram identificados como estudos associados à engenharia do conhecimento, à gestão do conhecimento e aos processos de aquisição, criação, representação, recuperação, compartilhamento, descoberta, combinação, similaridade e armazenamento do conhecimento.

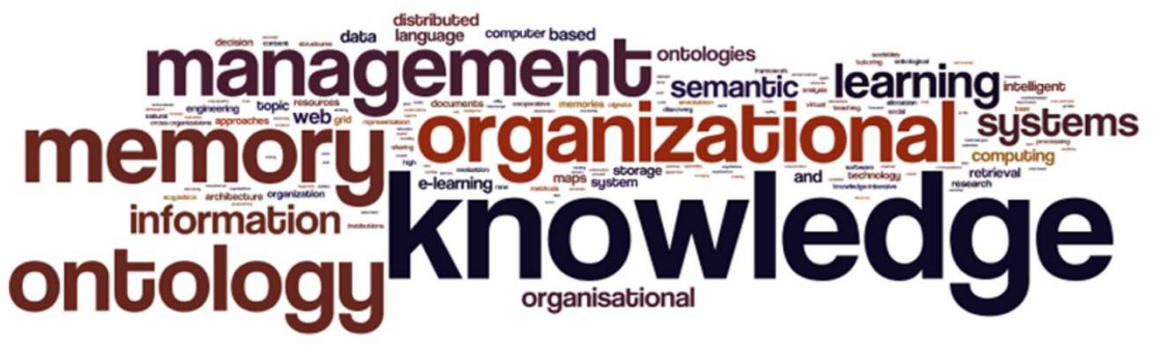

Figura 2 - Representação das palavras-chave utilizadas pelos trabalhos para artigos selecionados nas bases de dados WoS, Scopus e EBSCO para os termos "memória organizacional" e "ontologias"

Fonte: Dados da pesquisa.

Com as análises das palavras-chave e a leitura dos trabalhos, foi possível identificar os principais macrotemas (principais temáticas) tratados nesses estudos. A Figura 3 apresenta os macrotemas e os temas abordados. 


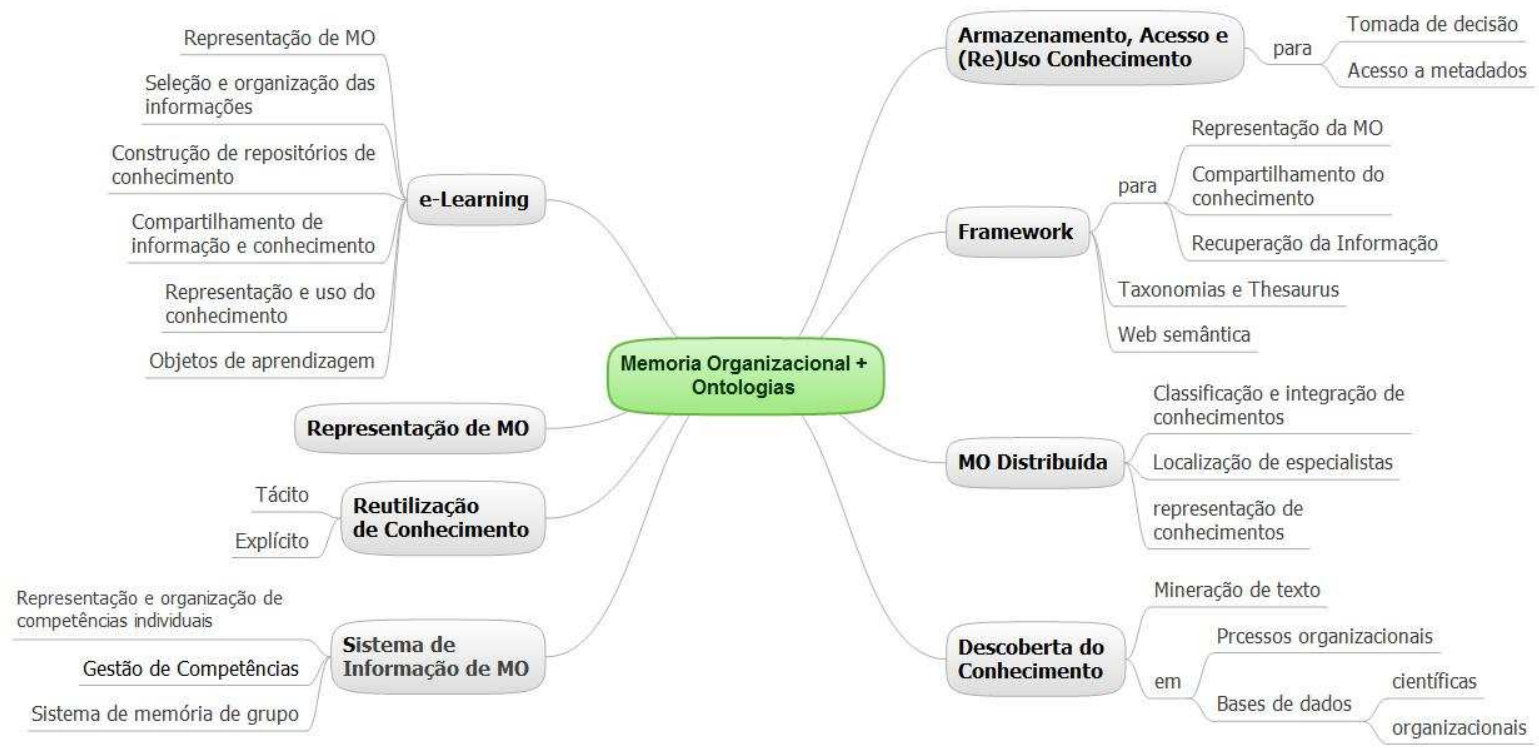

Figura 3 - Macrotemas e temas abordados pelos trabalhos para artigos selecionados nas bases de dados WoS, Scopus e EBSCO, para os termos "memória organizacional" e "ontologias".

Fonte: Dados da pesquisa.

Os artigos da temática e-Learning, por exemplo, discutem: a construção de repositórios de conhecimentos (como o fazem LAOUFI et al., 2011); a seleção e a organização das informações, por exemplo, o trabalho de Amrouche e colaboradores (2007); e o compartilhamento, a representação e o uso de informações e conhecimentos no contexto de projetos de educação a distância (e-Learning), como o artigo de Abel e outros (2003; 2004b), de Abel e colaboradores (2004a), de Zouaq, Frasson e Nkambou (2006a) e Zouaq, Nkambou e Frasson (2006b; 2007). Ainda nesse grupo, estão artigos que enfatizam a representação da memória organizacional baseada em ontologias (por exemplo, Abel, 2008) e os que enfocam objetos de aprendizagem, como o trabalho de Zouaq e Nkambou (2009).

O macrotema "representação da memória organizacional" caracteriza os trabalhos que tiveram como ênfase o desenvolvimento e a aplicação de ontologias para representar a memória de grupos e de organizações. Um trabalho representativo desse macrotema é o artigo de Ju (2006), no qual é apresentada uma arquitetura desenvolvida para apoiar a representação e a utilização da memória por meio de três tipos de ontologias: de domínio, de informação e enterpriseontology.

Os trabalhos que estão classificados no macrotema "reutilização de conhecimento", enfocam a utilização de ontologias para reutilizar conhecimentos tácitos (por exemplo, AYAZI; SHAMS, 2008) e explícitos (por exemplo, YANG; CHEN, 2006), que estão na memória organizacional. Outro grupo de trabalhos está enfocado em "sistemas de informação de memória organizacional" (organisationalmemoryinformation systems) e desenvolve tipos específicos de sistemas de apoio à memória, baseados 
em tecnologia da informação e ontologias. Um exemplo desses tipos de sistemas são os sistemas de memória de grupo para gestão de competências, a partir da representação e organização de competências individuais (como é proposto em DE VASCONCELOS; KIMBLE; ROCHA, 2003).

Na temática "armazenamento, acesso e (re)uso de conhecimentos", estão os trabalhos que enfocam a utilização de ontologias para facilitar o acesso, uso e reuso da memória organizacional (ALVARADO; ROMEROSALCEDO; SHEREMETOV, 2004), para a tomada de decisão (por exemplo, KALFOGLOU, 2007) e para o armazenamento e o acesso a metadados (por exemplo, KITOWSKI, 2008).

Em outro grupo estão os trabalhos que apresentaram frameworks para a representação da memória organizacional, por meio do uso de ontologias, taxonomias e tesauros, como o artigo de Anica-Popa (2011), ou, ainda, para apoiar o compartilhamento, como se dá em CarnpoyGomez (2004), a criação, por exemplo em Gao (2007), e a recuperação, como ocorre em Le, Dieng-Kuntz e Gandon (2004), de informações e conhecimentos.

No macrotema "memória organizacional distribuída", encontram-se os trabalhos que buscam estudar ontologias para representação, classificação e integração de conhecimentos que estão em diversos locais físicos ou virtuais (por exemplo, VAN ELST; ABECKER, 2002) e para localização de especialistas (WHITE; LUTTERS, 2007). Já no macrotema "descoberta de conhecimentos", encontram-se os artigos que enfocam, principalmente, aplicar ontologias e outras técnicas, tais como, mineração de texto (por exemplo, GULLA; SUGUMARAN, 2008), para localizar conhecimentos em processos de negócios (FURTADO; HAIMOWITZ; WURST, 2004) e em bases de dados científicas e organizacionais (FICENEC et al., 2003).

Foi possível identificar, a partir da análise das palavras-chave e da leitura dos trabalhos, que o principal contexto onde foram realizados os estudos sobre ontologias e memória organizacional foi no âmbito do eLearning.

\subsection{Principais referências citadas}

Das 845 referências citadas pelos trabalhos analisados, a obra de Abeckeret al. (1998) foi citada por oito dos 39 artigos analisados (ver Tabela 6). Essa obra desenvolveu um modelo para o processamento do conhecimento, na qual os autores argumentam que a memória organizacional pode ser muito mais do que um sistema de informações passivo, podendo ser um agente inteligente, computacional, que coopera com os humanos para a solução de problemas e que auxilia as empresas a transformarem a informação em ação. Para isso, o mencionado artigo aponta alguns requisitos cruciais para o sucesso da memória organizacional na prática industrial, tais como: coleta e organização sistemática de informações de várias fontes; integração dentro do 
ambiente de trabalho existente; preservação ativa da informação relevante; exploração do feedback do usuário para a manutenção e evolução do conhecimento organizacional; e minimização do up-front da engenharia do conhecimento, ou seja, o receio das organizações em investir tempo e dinheiro em uma tecnologia inovadora, cujos benefícios são incertos e perceptíveis a longo prazo.

Tabela 6 - As principais referências utilizadas nos artigos selecionados nas bases de dados WoS, Scopus e EBSCO para os termos "memória organizacional" e "ontologias"

\begin{tabular}{|c|c|c|c|c|c|}
\hline N. & Autores & Ano & $\begin{array}{l}\text { Titulo dos } \\
\text { trabalhos }\end{array}$ & Fonte da publicação & $\begin{array}{l}\text { Citaçõ } \\
\text { es }\end{array}$ \\
\hline 1 & $\begin{array}{l}\text { Abecker, A.; Bernardi, } \\
\text { A.; Hinkelmann, K.; } \\
\text { Kuhn, O.; Sintek, M. }\end{array}$ & 1998 & $\begin{array}{l}\text { Toward a technology } \\
\text { for organizational } \\
\text { memories }\end{array}$ & IEEE Intelligent Systems & 8 \\
\hline 2 & Gruber, T.R. & $\begin{array}{l}1993 \\
b\end{array}$ & $\begin{array}{l}\text { Toward Principles for } \\
\text { the Design of } \\
\text { Ontologies Used for } \\
\text { Knowledge Sharing }\end{array}$ & $\begin{array}{l}\text { International Journal of } \\
\text { Human-Computer Studies - } \\
\text { Special issue: the role of } \\
\text { formal ontology in the } \\
\text { information technology }\end{array}$ & 7 \\
\hline 3 & $\begin{array}{l}\text { Berners-Lee, T.; } \\
\text { Handler, J.; Lassila, } \\
\text { O.; }\end{array}$ & 2001 & The semantic web & Scientific American & 7 \\
\hline 4 & $\begin{array}{l}\text { Abel, M.H.; } \\
\text { Benayache, A.; Lenne, } \\
\text { D.; Moulin, C.; Barry, } \\
\text { C.; Chaput, B.; }\end{array}$ & 2004 & $\begin{array}{l}\text { Ontology-based } \\
\text { Organizational } \\
\text { Memory for e- } \\
\text { Learning }\end{array}$ & $\begin{array}{l}\text { Educational Technology \& } \\
\text { Society }\end{array}$ & 5 \\
\hline 5 & $\begin{array}{l}\text { Dieng, R.; Corby, O.; } \\
\text { Giboin, A.; Ribière, M.; }\end{array}$ & 1998 & $\begin{array}{l}\text { Methods and tools } \\
\text { for corporate } \\
\text { knowledge } \\
\text { management }\end{array}$ & $\begin{array}{l}\text { NRIA Sophia-Antipolis, } \\
\text { Project ACACIA }\end{array}$ & 5 \\
\hline 6 & $\begin{array}{l}\text { Staab, S.; Schnurr, } \\
\text { H.P.; Studer, R.; Sure, } \\
\text { Y. }\end{array}$ & 2000 & $\begin{array}{l}\text { Knowledge } \\
\text { Processes and } \\
\text { Ontologies }\end{array}$ & $\begin{array}{l}\text { Journal IEEE Intelligent } \\
\text { Systems }\end{array}$ & 5 \\
\hline
\end{tabular}

Fonte: Dados da pesquisa.

A segunda e a terceira referências mais citadas pelos trabalhos analisados foram os artigos de Gruber (1993b) e de Berners-Lee, Handler e Lassila (2001), ambos com sete citações cada. O primeiro trabalho aborda quais critérios de design para ontologias são necessários para compartilhar conhecimento e para a interoperabilidade entre programas baseados na conceitualização compartilhada; são eles: clareza, coerência, estendibilidade, viés de codificação mínima e compromisso ontológico mínimo. Gruber (1993b) discutiu o uso das ontologias em dois estudos de caso - no primeiro estudo, é abordada a representação de modelos matemáticos de sistemas de engenharia e, no segundo, é enfocado o compartilhamento de dados bibliográficos em bibliotecas escolares. Já o artigo de Berners-Lee, Handler e Lassila (2001) introduz o conceito de Web semântica, chamando a atenção para a importância de incorporar maior significado às páginas da Web. O argumento utilizado foi de que, por meio da inserção de metadados legíveis por máquina, é possível automatizar e tornar mais inteligente o acesso às páginas da Web. 
O artigo de Abel e colaboradores (2004a), citado por cinco trabalhos analisados, analisa como a ontologia pode ser utilizada para a memória de documentos adaptados particularmente ao contexto do e-Learning. A fim de organizar os recursos referentes ao aprendizado por meio da memória organizacional, os autores mencionados utilizaram dois tipos de ontologias: a primeira, genérica, concentrou-se no treinamento de forma ampla; e a segunda foi relacionada com a aplicação do domínio específico, ou seja, um programa de treinamento em específico. Os mesmos autores apresentaram abordagens para a construção de ontologias e como elas podem ser trabalhadas conjuntamente.

O artigo de Dienget al. (1998) - também citado por cinco trabalhos analisados - evidencia métodos, técnicas e ferramentas para gerir o conhecimento, a partir da memória corporativa. Os autores analisaram problemas e soluções do ciclo de vida da memória corporativa: descoberta de necessidades, construção, difusão (especialmente por meio da internet), uso, avaliação e evolução. Eles concluíram que pesquisas referentes à memória corporativa requerem uma abordagem multidisciplinar e, na sequência, apresentaram várias questões de pesquisa relacionadas ao ciclo de vida, que podem ser respondidas antes de se constituir a memória corporativa em uma empresa.

Por último, também citado por cinco trabalhos, o artigo de Staab e outros (2000) aborda, pelo menos, duas dimensões do processo de conhecimento que necessitam ser organizadas. A primeira trata do "processo de metaconhecimento", necessário para introduzir as soluções de gestão do conhecimento dentro de uma empresa (nesse processo, ontologias são desenvolvidas agindo como "aglutinador" entre os diferentes processos de conhecimento); e a segunda aborda os processos de conhecimento que eram realizadas durante a execução de soluções de gestão do conhecimento.

Por meio das análises das referências citadas pelos 39 trabalhos analisados, identificou-se que todos os principais artigos mencionados pelos autores abordam, de alguma maneira, a utilização de ontologias associada à memória organizacional. Com isso, pode-se dizer que os estudos analisados, que buscam estabelecer algum tipo de interface entre os dois temas, utilizam como principais fundamentos teóricos outros trabalhos que, direta ou indiretamente, aplicaram ontologias para a solução de um determinado problema de memória organizacional.

\section{Considerações finais}

Este artigo apresentou os resultados de um estudo bibliométrico sobre os termos "memória organizacional" e "ontologias". Ele foi desenvolvido com o propósito de disponibilizar aos pesquisadores e interessados nos temas, um mapeamento sobre o perfil e as características das pesquisas que tratam dos assuntos abordados.

Nesse sentido, o estudo utilizou técnicas bibliométricas aplicadas para a captura de dados que, contextualizados, possibilitaram identificar e 
informar aos interessados sobre os padrões e as tendências da literatura científica e mostrou, assim, que pesquisas nessa área podem ser promissoras por auxiliarem os pesquisadores na identificação dos basilares teóricos em determinada área de estudo. Essas técnicas permitiram lidar com o desafio de juntar informações de três diferentes bases de dados, traçando um perfil representativo do campo de estudo da memória organizacional e ontologias.

Devido ao fato da pesquisa bibliométrica realizada abarcar artigos disponíveis em três bases de dados (EBSCO, Scopus e WoS), diferentes padrões de cadastro e ausência de informações dos artigos foram identificados. Sempre que isso aconteceu, os pesquisadores buscaram localizar as informações complementares que faltavam. Entretanto, as principais limitações deste trabalho estão associadas ao processo de localização e seleção dos artigos analisados. Ou seja, a primeira busca nas bases de dados (sem considerar os trabalhos que se repetiam em mais de uma base), possibilitou localizar 52 trabalhos, porém foram retirados 13 (artigos), porque não tinham texto completo disponível e/ou informações sobre os seus autores, restando, assim, 39 trabalhos para serem analisados. Todavia, a análise desses trabalhos possibilitou traçar um mapa sobre as pesquisas que tratam da memória organizacional e das ontologias de modo conjunto. Também, foi possível analisar o desenvolvimento desse enfoque de pesquisa no decorrer do tempo, as características e as formas de disseminação e (re)utilização de conhecimentos acadêmico-científicos produzidos por vários pesquisadores, espalhados por diversos países.

Por meio deste estudo, identificou-se que o "plano de fundo" da área é o e-Learning, destacando-se as pesquisas coordenadas por Marie-Hélène Abel, no projeto MEMORAe. Esse projeto utilizou o e-Learning para cursos ou treinamento de diversos sujeitos (alunos, professores, designers instrucionais, administradores, etc.), para gestão dos recursos (exercícios, estudos de caso, etc.) em diferentes formatos (relatórios, livros, sites, etc.) nas instituições de ensino. Os recursos, conhecimentos explícitos e competências formais foram geridos por meio de uma ontologia denominada de "aprendizagem da memória organizacional".

A memória organizacional pode ser entendida como uma área que faz a intersecção com outras áreas de pesquisa, tais como gestão do conhecimento, aprendizagem organizacional e engenharia do conhecimento. Dentre essas intersecções, estão os trabalhos que estudam as técnicas da inteligência artificial, como, por exemplo, as ontologias que têm sido desenvolvidas e aplicadas para a compreensão, o uso e o reuso do conhecimento para a tomada de decisão nas organizações.

Em suma, o mapeamento do campo de pesquisas sobre memória organizacional e ontologias, apresentado neste trabalho, promove uma maior compreensão sobre o histórico e o estado atual desse campo de pesquisa no nível internacional, a partir dos dados bibliográficos recuperados nas três diferentes bases de dados científicas. Entre outras informações, este artigo - ao apresentar sinteticamente as principais 
fontes de publicações com maior quantidade de trabalhos sobre memória organizacional e ontologias - aponta possíveis caminhos para que os pesquisadores saibam por onde começar ou aprofundar as suas pesquisas a respeito dos dois temas interligados e, também, conheçam quais os trabalhos que têm fundamentado as pesquisas nessa área.

Portanto, como é possível perceber, o estudo gera várias possibilidades de futuras pesquisas e contribui para uma visão ampla e global do quadro das bibliografias da memória organizacional e das ontologias. Além disso, fornece vários insumos para enriquecer a discussão sobre o rumo das pesquisas e as prováveis tendências nesse campo de pesquisa.

\section{Referências}

ABECKER, A. et al. Toward a technology for organizational memories.IEEE Intelligent Systems,v. 13, p. 40-48, 1998.

ABEL, M. H. et al. Gestion des ressources pédagogiques d'une eformation. Document Numerique, v. 7, n. 1-2, p. 111-128, 2003.

ABEL, M. H. et al.Ontology-based organizational memory for e-learning. Educational Technology and Society, v. 7, n. 4, p. 98-111, 2004a.

$A B E L, M . H$. et al. Using two ontologies to index e-learning resources. In: THE 2004 IEEE/WIC/ACM INTERNATIONAL CONFERENCE ON WEB INTELLIGENCE - WI 2004, 1., 2004, Beijing. Anais.... Beijing: IEEE, 2004. p. 549-552.

ABEL, M. H. Competencies management and learning organizational memory. Journal of Knowledge Management, v. 12, n. 6, p. 15-30, 2008.

ALMEIDA, E. C. E. de. O portal de periódicos da Capes: estudo sobre a sua evolução e utilização. 2006. 175f. Dissertação (Mestrado) - Mestrado em Desenvolvimento Sustentável, Universidade de Brasília, Brasilia, 2006.

ALVARADO, M.; ROMERO-SALCEDO, M.; SHEREMETOV, L. A corporative memory based on the user profiles. Expert Systems with Applications, v. 26, n. 1, p. 87-94, 2004.

AMROUCHE, H. et al.Interrogation of ontologies formalized in topic maps with TMQL in e-learning context. In:ATLANTIC WEB INTELLIGENCE CONFERENCE - AWIC'2007, 5., 2007, Fontainbleau. Proceedings... Fontainbleau, France, June, 2007., v. 43 p. 29-34.

ANDERSON, M.H.; SUN, P.Y.T. What have scholars retrieved from Walsh and Ungson (1991)? A citation context study.Management Learning, v. 41, n. 2, p. 131-145, 2010.

ANICA-POPA, L.-E.OMCCAAF conceptualizing of taxonomy and thesaurus construction. Annals of DAAAM \& Proceedings, p. 913-914, 2011.

AYAZI, F.; SHAMS, F. Using ontologies for improvement of tacit knowledge. In: INFORMATION AND COMMUNICATION TECHNOLOGIES: 
FROM THEORY TO APPLICATIONS, 3., 2008, Tehran. Anais... Tehran: ICTTA, 2008. p. 1-4.

BALANCIERI, R. Análise de redes de pesquisa em uma plataforma de gestão em ciência e tecnologia: uma aplicação à plataforma Lattes. 2004. 117f. Dissertação (Mestrado) - Curso de Programa de Pós-graduação em Engenharia de Produção, Universidade Federal de Santa Catarina - UFSC, Florianópolis, 2004.

BERNERS-LEE, T.; HENDLER, J.; LASSILA, O. The Semantic Web. Scientific American, p. 35-43, 2001.

BORST, W. N. Construction of engineering ontologies for knowledge sharing and reuse. 1997. 231f. Tese (Doutorado) - Curso de Electrical Engineering, Mathematics And Computer Science (EEMCS), University Of Twente, Enschede, 1997.

BRAMBILLA, S. D. S.; STUMPF, I. R. C. Produção científica da UFRGS representada na WOS (2000-2009). Perspectivas em Ciência da Informação, v.17, n.3, p.34-50, jul./set. 2012.

CARDENAS, J. M. Knowledge or memory: what is the right choice about Information Technology concerns? In: TECHNOLOGY MANAGEMENT FOR GLOBAL ECONOMIC GROWTH (PICMET), 10., 2010, São

Paulo. Anais... São Paulo: Proceedings Of Picmet, 2010. p. 1-4.

CARNPOY-GOMEZ, L. Constructing natural knowledge ontologies to implement semantic organizational memory.In: MEZIANE, F.; METAIS, E. (Ed.).Natural language processing and information systems. Berlin: Springer-VerlagBerlin, 2004. v. 3136, p. 354-361.

CROSSAN, M. M.; APAYDIN, M. A multi-dimensional framework of organizational innovation: a systematic review of the literature.Journal of Management Studies, v. 47, n. 6, p. 1154-1191, 2010.

DE VASCONCELOS, J. B.; KIMBLE, C. ; ROCHA, Á. Organisational memory information systems an example of a group memory system for the management of group competencies.Journalof Universal Computer Science, v. 9, n. 12, p. 1410-1427, 2003.

DIAS, E. A. V.; COSTA, H. G. Levantamento bibliométrico no âmbito da ontologia. In: CONGRESSO NACIONAL DE EXCELÊNCIA EM GESTÃO, 7., 2011, Rio de Janeiro. Anais... Rio de Janeiro: Sistema Firjan, 2011. p. 120.

DIENG, R. Methods and tools for corporate knowledge management. International Journal Of Human-computer Studies, [S. I.], v. 51, n. 1, p.567-598, set. 1999.

FICENEC, D. et al. Computational knowledge integration in biopharmaceutical research.Briefings in bioinformatics, v. 4, n. 3, p. 260278, 2003. 
FURTADO, J. J. V.; HAIMOWITZ, I.; WURST, M. Knowledge discovery in databases and agent-mediated knowledge management. Lecture Notes In Computer Science, [s. I.], v. 2956, n. 1, p. 339-350, abr. 2004.

GAO, J. Supporting creative organizational learning: Knowledge applicability management based on task circumstance.JisuanjiXuebao/Chinese Journal of Computers, v. 30, n. 9, p. 1533-1543, 2007.

GRUBER, T. R. A translation approach to portable ontology specification.Knowledge Acquisition, v. 5, n. 2, p. 199-220, 1993 a.

GRUBER, T. R. Toward Principles for the Design of Ontologies Used for Knowledge Sharing. Padova: Knowledge Systems Laboratory, 1993. 23 p.

GRUBER, T. R. Toward principles for the design of ontologies used for knowledge sharing? International Journal of Human Computer Studies, London, v.43, n. 5-6, p. 907-928, 1995.

GUARINO, N. Formal ontology, conceptual analysis and knowledge representation.InternationalJournalofHuman-Computer Studies, v. 43, n. 5-6, p. 625-640, 1995.

GUEDES, V.; BORSCHIVER, S. Bibliometria: uma ferramenta estatística para a gestão da informação e do conhecimento, em sistemas de informação, de comunicação e de avaliação científica e tecnológica. In: ENCONTRO NACIONALDE CIÊNCIA DA INFORMAÇÃO, 6., 2005, Salvador. Anais... Salvador: CINFORM, 2005. p. 1-18.

GULLA, J. A.; SUGUMARAN, V. An interactive ontology learning workbench for non-experts. In: INTERNATIONAL WORKSHOP ON ONTOLOGIES AND INFORMATION SYSTEMS FOR THE SEMANTIC WEB, 2., 2008, New York. Proceedings... New York: ONISW, 2008. p. 9-16.

HERCULANO, R. D.; NORBERTO, A. M. Q. Análise da produtividade científica dos docentes da Universidade Estadual Paulista, campus de Marília/SP. Perspectivas em Ciência da Informação, v.17, n.2, p.57-70, abr./jun. 2012.

JU, T. L. An open architecture for codifying organizational memory. In: INTERNATIONAL CONFERENCE ON EDUCATION AND INFORMATION SYSTEMS, TECHNOLOGIES AND APPLICATIONS: EISTA 2006, 4., 2006, Orlando. Anais... Orlando: EISTA, 2006. p. 1-6.

KALFOGLOU, Y. Using ontologies to support and critique decisions. Engineering Intelligent Systems, v. 15, n. 3, p. 159-166, 2007.

KANKANHALLI, A; TAN, B; WEI, K.Contributing knowledge to electronic knowledge repositories: An empirical investigation.MIS Quarterly, v. 29, n. 1, p. 113-143, 2005.

KITOWSKI, J. Semantic approach to capability and capacity computing. In: INTERNATIONAL SYMPOSIUM ON PARALLEL AND DISTRIBUTED COMPUTING, 7., 2008, Krakow. Anais... Krakow: ISPDC, 2008. p. 1 - 20. 
KOBASHI, N. Y.; SANTOS, R. N. M. Institucionalização da pesquisa científica no Brasil: cartografia temática e de redes sociais por meio de técnicas bibliométricas. TransInformação, v. 18, n. 1, p. 27-36, jan./abr. 2006.

LAOUFI, A. et al. An ontology based architecture to support the knowledge management in higher education. In: INTERNATIONAL CONFERENCE ON MULTIMEDIA COMPUTING AND SYSTEMS (ICMCS), 1., 2011, Ouarzazate, Morocos.Anais... . Ouarzazate, Morocos: IEEE, 2011. p. 1-6.

LE, B. T.; DIENG-KUNTZ, R.; GANDON, F.On ontology matching problems - For building a corporate semantic web in a multi-communities organization. In: SERUCA, I.; FILIPE, J., et al, Porto. p.236-243, 2004.

LEHNER, F.; MAIER, R.K. How can organizational memory theories contribute to organizational memory systems? Information Systems Frontiers, v. 2, n. 3/4, p. 277- 298, 2000.

LOPES, S. et al.A bibliometria e a avaliação da produção científica: indicadores e ferramentas. Actas dos Congressos Nacionais de Bibliotecários, Arquivistas e Documentalistas, n. 11, 2012. Disponível em: <http://www.bad.pt/publicacoes/index.php/congressosbad/issue/view/10 >. Acesso em: 23 nov. 2012.

MACIAS-CHAPULA, C. A. O papel da informetria e da cienciometria e sua perspectiva nacional e internacional. Ciência da informação, v. 27, n. 2, p. 134-140, 1998.

MELGAR SASIETA, H.; BePPleR, A.; PACHECO, R. C. dos S. A Memória organizacional no contexto da engenharia do

conhecimento. Datagramazero, Rio de Janeiro, v. 12, n. 4, p.1-17, ago. 2011. Disponível em: <http://www.dgz.org.br/ago11/Art_06.htm>. Acesso em: 23 jan. 2013.

MESQUITA, R.et al. Elaboração e aplicação de instrumentos para avaliação da base de dados Scopus. PerspectivasemCiência da Informação, v. 11, n. 2, p. 187-205, 2006.

MUSEN, M. A. Medical informatics: searching for underlying components. Methods of Information in Medicine, v. 41, n.1, p.12-19, 2002.

NAKATA, K. Knowledge as a social medium. New Generation Computing, v. 17, n. 4, p. 395-405, 1999.

NEVO, D. ; WAND, Y. Organizational memory information systems: a transactive memory approach. DecisionSupport Systems, v.39, n.4, p.549-562, 2005.

OLIVEIRA, E.; GRACIO, M. C. Indicadores bibliométricos em ciência da informação: análise dos pesquisadores mais produtivos no tema estudos métricos na base Scopus. PerspectivasemCiência da Informação, v.16, n.4 p. 16-28, 2011. 
OLIVERA, F. Memory systems in organizations: an empirical investigation of mechanisms for knowledge collection, storage and access. Journal of Management Studies, v. 37, n. 6, p. 811-832, 2000.

PENCIUC, D. ; ABEL, M.-h; ABEELE, D. V. D. Support for collaborative development of a customer solution in railway transport. In: COMPUTER SUPPORTED COOPERATIVE WORK IN DESIGN (CSCWD), 15., 2011, Lausanne. Anais... Lausanne: CSCWD, 2011. p. 544-550.

RAUTENBERG, S.; TODESCO, J. L.; GAUTHIER, F. A. O. Processo de desenvolvimento de ontologias: uma proposta e uma ferramenta. Rev. Tecnol., v.30, p. 133-144, 2009.

SANTOS, J. L. S.; URIONA-MALDONADO, M.; SANTOS, R. N. M. dos. Mapeamento das publicações acadêmico-científicas sobre memória organizacional. In: ENCONTRO NACIONAL DA ASSOCIAÇÃO NACIONAL DE PÓS-GRADUAÇÃO E PESQUISA EM ADMINISTRAÇÃO, 35., 2011, Rio de Janeiro. Anais... Rio de Janeiro: Enanpad, 2011. p. 1-17.

STAAB, S.et al. Knowledge processes and ontologies. Journal IEEE Intelligent Systems, p.25-33, 2000.

STEIL, A.; SANTOS, J.L.S. Building conceptual relations between organizational learning, knowledge, and memory.International Journal of Business and Management Tomorrow, v. 2, n. 2, p. 1-9, 2012.

STEIN, E. Organizational memory: review of concepts and recommendations for management. International Journal of Information Management, v. 15, n.2, p. 17-32, 1995.

TOLEDO, C. M. et al.An ontology-driven document retrieval strategy for organizational knowledge management systems.Electronic Notes in Theoretical Computer Science, v. 281, p. 21-34, 2011.

USCHOLD, M; GRUNINGER, M. Ontologies: principles, methods and applications. Knowledge Engineering Review, New York, v.11, n. 2, p. 93136, 1996.

VALASKI, J.; MALUCELLI, A.; REINEHR, S. Ontologies application in organizational learning: A literature review. Expert Systems with Applications, v. 39, p. 7555-7561, 2012.

VAN ELST, L.; ABECKER, A. Ontologies for information management: balancing formality, stability, and sharing scope. Expert Systems with Applications, v. 23, n. 4, p. 357-366, Nov. 2002.

WALSH, J.P.; UNGSON, G.R. Organizational memory.The Academy of Management Revie, v. 16, n. 1, p. 57-91, 1991.

WEINBERGER, H.; TE'ENI, D. ; FRANK, AJ. Ontology-based evaluation of organizational memory.Journal of the American Society for Information Science and Technology, v. 59, n. 9, p. 1454-1468, 2008.

WHITE, K. F.; LUTTERS, W. G. Structuring cross-organizational knowledge sharing. In: PROCEEDINGS OF THE 2007 INTERNATIONAL ACM 
CONFERENCE ON SUPPORTING GROUP WORK, 1., 2007, Sanibel Island. Proceedings... Sanibel Island: ACM, 2007. p. 187-196.

YANG, K.-J.; CHEN, Y.-M. Ontology-based knowledge retrieval in organizational memory. In: INTERNATIONAL CONFERENCE ON INNOVATIVE COMPUTING, INFORMATION AND CONTROL, 1., 2006, Beijing. Anais... Beijing: IEEE, 2006. p. 566-569.

ZOUAQ, A.; FRASSON, C.; NKAMBOU, R. An ontology-based solution for knowledge management and eLearning integration. In: IKEDA, M. et al.(Ed.). Intelligent Tutoring Systems. Berlin: Springer-Verlag Berlin, 2006a. v.4053, p. 716-718.

ZOUAQ, A.; NKAMBOU, R.; FRASSON, C. The knowledge puzzle: an integrated approach of intelligent tutoring systems and knowledge management. In: INTERNATIONAL CONFERENCE ON TOOLS WITH ARTIFICIAL INTELLIGENCE, 18., 2006, Washington. Anais... Washington: IEEE, 2006. p. 575-582.

ZOUAQ, A.; NKAMBOU, R.; FRASSON, C. An integrated approach for automatic aggregation of learning knowledge objects. Interdisciplinary Journal of Knowledge \& Learning Objects, v. 3, p. 135-162, 2007.

ZOUAQ, A. ; NKAMBOU, R. Enhancing learning objects with an ontologybased memory.IEEE Transactions on Knowledge and Data Engineering, v. 21, n. 6, p. 881-893, 2009.

* Caso o leitor tenha interesse em conhecer as referências dos 39 artigos analisados nesta pesquisa bibliométrica poderá entrar em contato com os autores. 